\section{Identification of a Safe Site for Percutaneous Endoscopic Gastrostomy Placement in Patients with Marked Bowel Distension: May Octreotide Have a Role?}

Percutaneous endoscopic gastrostomy (PEG) is considered the procedure of choice for long-term enteral feeding of patients unable to swallow. Sometimes it is also used to achieve gastric decompression in small-bowel obstruction due to diffuse abdominal carcinomatosis (1). An absolute contraindication to PEG is an inability to obtain an appropriate apposition between the anterior wall of the stomach and the abdominal wall (2). This condition may occur frequently in patients with intestinal obstruction, owing to the interposition of distended bowel loops between the stomach and abdominal wall.
Somatostatin and its analogues have been shown to have a powerful inhibitory action on gastrointestinal endocrine and exocrine secretions (3), and to significantly reduce bowel distension in mechanical intestinal obstruction (4). We report here on a case in which the administration of octreotide, an analogue of somatostatin with a longer half-life and higher potency (3), made it possible to obtain an appropriate site for PEG placement in the presence of marked and diffuse bowel distension, which seemed to contraindicate the procedure.

A 64-year-old man, who had undergone anterior resection and sigmoidostomy for rectum cancer four years previously, was admitted to our Department for diffuse carcinomatosis with multiple sites of obstruction. Repeated episodes of vomiting occurred over the following days, and a nasogastric tube was placed. The vomiting ceased, but the tube created great discomfort, and the patient gave 
his consent to PEG placement. Despite the nasogastric drainage of $1500-2000 \mathrm{ml} /$ day, a preliminary ultrasound examination of the abdomen showed marked and diffuse bowel distension in all quadrants of the abdomen, including the epigastrium and the left upper periumbilical area. The identification of a safe site for PEG placement using transillumination of abdominal wall was not possible, and the procedure was suspended. Subcutaneous octreotide administration $(0.1 \mathrm{mg} / 8 \mathrm{~h})$ was started, and after two days nasogastric drainage dropped to $300-350 \mathrm{ml} /$ day. The following day, the ultrasound examination of the abdomen was repeated, and showed a remarkable reduction of the bowel distension. PEG placement was tried again: transillumination of the abdominal wall was possible, finger palpation of the transilluminated area was clearly seen by the endoscopist, and a PEG was placed without complications. At present (4 January, 1994), tue patient is still alive, 71 days after the procedure.

In small-bowel obstruction, bowel distension due to accumulated gastric, pancreatic, biliary, and intestinal secretions is a powerful stimulus for further intestinal secretion, which provokes further bowel distension (5). In our patient, this vicious cycle resulted in enormous bowel distension, making PEG placement impossible. Somatostatin and its analogue octreotide, due to their inhibitory action on gut secretions, can positively intervene in this vicious cycle, and may have a place in the management of patients with bowel obstruction in whom surgery is not indicated (5). Indeed, somatostatin-treated patients with mechanical small-bowel obstruction have been reported to have significantly less bowel distension than placebo-treated ones (4), and it is very likely that in our patient octreotide played a key role in reducing the bowel distension, making the identification of a safe site for PEG placement possible.
In conclusion, we think that our experience, though anecdotal, can be summarized in a suggestion: a two or three-day long administration of octreotide or other somatostatin analogues should always be attempted before abandoning attempts at PEG placement in patients with bowel obstruction.

S. Sartori ${ }^{1}$, L. Trevisani ${ }^{1}$, I. Nielsen ${ }^{\text {}}$, D. Tassinari ${ }^{\text {I }}$, E. Righini ${ }^{2}$ ${ }^{1}$ Department of Medicine and Oncology Service, Ospedale S. Anna, Ferrara, italy; ${ }^{2}$ Istituto di Anestesiologia e Rianimazione, University of Ferrara, Italy

\section{References}

1. Shearen JG, Goudale RL, Himmelein S: Percutaneous endoscopic gastrostomy. Minn Med 1985; 69: 189-191.

2. Foutch PG, Talbert RN, Waring JP et al.: Percutaneous endoscopic gastrostomy in patients with prior abdominal surgery: virtues of the safe tract. Am J Gastroenterol 1988; 83: 147-150.

3. Gyr KE, Meier R: Pharmacodynamic effects of Sandostatin in the gastrointestinal tract. Digestion 1993; 54 (suppl 1): 14-19.

4. Gastounis E, Hadjinikolau L. Ioannou $N$ et al.: Somatostatin as adjuvant therapy in the management of obstructive ileus. Hepatogastroenterology 1989; 36: 538-539.

5. Mulvihill SJ: Perioperative use of octreotide in gastrointestinal surgery. Digestion 1993; 54 (suppl 1): 33-37.

Corresponding Author

S. Sartori, M.D., II Divisione Medica, Ospedale S. Anna, corso Giovecca 203, 44100 Ferrara, Italy 